\title{
A study of gas-particle partitioning of PAH according to adsorptive models and season
}

\author{
S. Pongpiachan ${ }^{1,2}$, K. F. Ho ${ }^{3}$ \& S. C. Lee ${ }^{3}$ \\ ${ }^{1}$ Faculty of Environmental Management, Prince of Songkhla University, \\ Thailand \\ ${ }^{2}$ National Center of Excellence for Environmental and Hazardous Waste \\ Management-PSU Satellite Center, Prince of Songkhla University, \\ Thailand \\ ${ }^{3}$ Research Center for Urban Environmental Technology \& Management, \\ Department of Civil \& Structural Engineering, \\ The Hong Kong Polytechnic University, China
}

\begin{abstract}
Vapour-phase and particulate air samples were collected in a rural area of England in a cold period (20/11/2003 - 22/12/2003) and a warm period (5/5/2004 - 26/5/2004) to investigate the seasonal effect on gas-particle partitioning of PAHs. Three different sorptive models, namely Dachs-Eisenreich (ab/adsorption), $\mathrm{K}_{\mathrm{OA}}$ (absorption into organic layer) and $\mathrm{K}_{\mathrm{SA}}$ (adsorption on the soot surface) were applied and, as expected, the inclusion of an adsorptive mechanism provided better predictions of $\mathrm{K}_{\mathrm{P}}$ values for all PAHs. The ratios of measured- $\mathrm{K}_{\mathrm{P}} /$ Dachs-Eisenreich- $\mathrm{K}_{\mathrm{P}}$ are $1.03 \pm 0.12$ and $1.06 \pm 0.15$ in the cold period and warm period respectively for a group of medium molecular weight compounds. The model results indicate a far greater importance for both adsorptive and absorption processes rather than the absorption mechanism alone in determining the gas-particle partitioning of PAH. There was little difference between $\log \mathrm{K}_{\mathrm{P}}$ values for the medium and high molecular weight PAH between the warm period and cold period campaigns despite a difference in mean temperature of $10^{\circ} \mathrm{C}$. This can be explained by a difference in aerosol properties between the two campaigns and it is postulated to be attributable in minor part to a greater fraction of elemental carbon present in the warm period aerosol, which derives from a greater proportion of continental air masses than in the cold period, but predominantly to the lesser relative humidity in the warm period
\end{abstract}


leading to lower hygroscopic growth of internally-mixed aerosol and consequently less blockage of adsorption sites by liquid water.

Keywords: polycyclic aromatic hydrocarbons, organic carbon, elemental carbon, gas-particle partitioning coefficient, absorption, adsorption, seasonal variation.

\section{Introduction}

Polycyclic aromatic hydrocarbons are ubiquitous pollutants in the atmospheric environment, generally containing from two to eight benzene rings. They can be produced in natural and anthropogenic processes [1]. Many PAHs have received much attention because some of these compounds are considered to be toxic, carcinogenic and/or mutagenic [2]. Many studies have reported that gas/particle partitioning is an important inter-media exchange process in controlling the atmospheric behavior, transport and transformation of PAHs [3, 4]. Investigating the gas-particle partitioning is not only useful for predicting the fate of PAHs in the atmospheric environment but also crucial for assessing respiratory health effects since the risk deriving from inhaling these compounds depends in part on whether they exist in the gaseous or particulate phase. In addition, the gasparticle partitioning coefficient is a key factor influencing the global distribution of PAHs via long range transport. Two different mechanisms have been employed to describe the gas-particle partitioning of PAHs, namely physical adsorption onto the aerosol surface and chemical absorption into the aerosol organic layer [5]. Both mechanisms lead to a linear relationship between $\log K_{\mathrm{P}}$ and $\log P_{\mathrm{L}}{ }^{\circ}$ (Junge-Pankow Sorption Model), where $P_{\mathrm{L}}{ }^{\circ}$ is the compound's subcooled liquid vapor pressure and $K_{\mathrm{p}}$ is the gas particle partitioning coefficient.

During the past few years, an intense research effort has been made to develop the modeling framework for the sorption processes in order to predict the gas/particle partitioning of PAHs. Fenizio et al. [6] proposed a method to predict $K_{\mathrm{p}}$ values of a wide range PAHs by using only the octanol/air partition coefficient $\left(K_{\mathrm{OA}}\right)$ assuming that absorption is the main sorption process [6].

$$
K_{P}=\frac{f_{O M} M W_{O C T} \gamma_{O C T}}{\rho_{O C T} M W_{O M} \gamma_{O M} 10^{12}} K_{O A}
$$

where $\rho_{\text {oct }}\left(0.824 \mathrm{~kg} \mathrm{~L}^{-1}\right.$ at $\left.20^{\circ} \mathrm{C}\right)$ is the density of the octanol and yare the activity coefficients of the PAHs in organic matter (OM) and octanol (oct), respectively. In contrast to $\mathrm{OM}$ partitioning, several researchers are concerned about a prominent role of elemental carbon (EC) adsorption in the atmosphere. Dachs and Eisenreich [5] reported the evidence for adsorption to EC is dominating the gas-particle partitioning in the New Jersey atmosphere. Assuming that EC is a surrogate for the soot phase, the overall gas-particle partitioning coefficient that accounts for both the organic matter and the soot phases is given by

$$
K_{P}=\frac{f_{O M} M W_{O C T} \gamma_{O C T}}{\rho_{O C T} M W_{O M} \gamma_{O M} 10^{12}} K_{O A}+f_{E C} \frac{a_{E C}}{10^{12} a_{S o o t} \rho_{E C}} K_{S A}
$$


where $f_{\mathrm{EC}}$ is the fraction of elemental carbon in the aerosol, $a_{\mathrm{Soot}}$ is the surface area of the soot used to determine $K_{\mathrm{SA}}, a_{\mathrm{EC}}$ is the specific surface area of the elemental carbon, $\rho_{\mathrm{EC}}$ is the density of elemental carbon and $K_{\mathrm{SA}}$ is the soot-air partitioning coefficient. When the partitioning is determined by both $a d$ sorptive and $a b$ sorptive processes, $K_{\mathrm{p}}$ can be accurately predicted by using the DachsEisenreich model (Eqn (2)). Recently, Odabasi et al. [7] determined octanol-air partition coefficients $\left(K_{\mathrm{OA}}\right)$ and super-cooled liquid vapor pressure $\left(P_{\mathrm{L}}{ }^{\circ}\right)$ for 13 PAHs as a function of temperature. In the present work, we compared the gasparticle partitioning coefficients $\left(K_{\mathrm{P}}\right)$ of PAHs estimated by using the DachsEisenreich gas-particle partitioning model, the $K_{\mathrm{OA}}$ model and the $K_{\mathrm{SA}}$ model with the measured $K_{\mathrm{P}}$ values of PAHs collected at a rural site (Whitbourne, UK). In order to clarify the controlling factors for the accuracy of each model, we evaluated the effect of OM conversion factor, specific surface areas of various types of EC, seasons (i.e. cold period and warm period) and molecular weight (MW) (i.e. low, medium and high MW) of PAHs, on the variation of $K_{\mathrm{P}}$ values.

\section{Methodology}

\subsection{Sampling sites and descriptions}

Air samples were collected at Whitbourne (Lat: 52:12:29 N, Long: 2:24:41 W) in a rural agricultural area of the UK, located $8 \mathrm{~km}$ northwest of the city of Worcester (Figure 1). $\mathrm{PM}_{10}$ samples were collected during the periods from $20 / 11 / 2003$ to $22 / 12 / 2003$ (cold period-period) and from 5/5/2004 to 26/5/2004 (warm period-period). Using a Graseby-Andersen high volume air sampler with $\mathrm{PM}_{10}$ TE-6001 inlet, $24 \mathrm{~h}$ sampling was carried out every day started at $6 \mathrm{pm}$ and stopped at $6 \mathrm{pm}$ of another day.

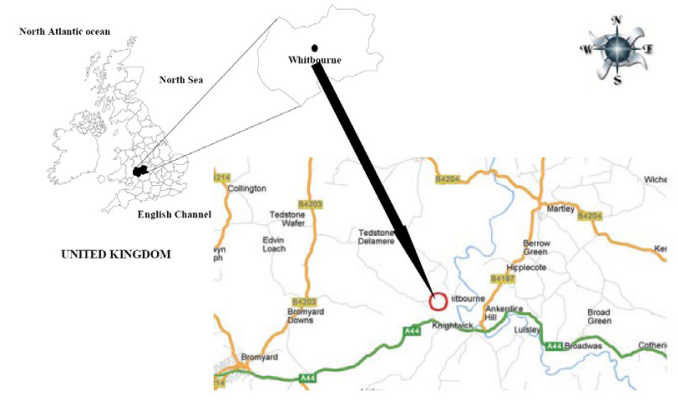

Figure 1: Location of air sampling site for this study $(\sim 33 \mathrm{~km}$ southwest of Birmingham city centre and $\sim 8 \mathrm{~km}$ northwest of Worcester city centre). 


\subsection{Sample analysis}

\subsubsection{PAHs analysis}

2.2.1.1 Sample extraction All solvents were HPLC grade, purchased from Fisher Scientific. The EPA 16 PAH surrogate cocktail in methanol $\left(100 \mu \mathrm{g} \mathrm{ml}^{-1}\right)$ used as native standard was obtained from Greyhound, whereas the additional deuterated PAH used as internal standards were supplied by Chiron AS. Soxhlet thimbles and glass fiber filters were obtained from Whatman (Maidstone, UK). All materials used (silica gel, glass and cotton wool, QFFs etc.) were Soxhlet extracted with DCM for $24 \mathrm{~h}$, and kept dry (in desiccators) until use. All glassware was cleaned by washing with distilled water before drying at $55^{\circ} \mathrm{C}$, and rinsed with DCM just before use. The extraction of PAHs was conducted using two different sizes of Soxhlet extractors (i.e. $250 \mathrm{ml}$ for QFFs and 1-litre for PUF plug).

2.2.1.2 Fractionation/cleanup and blow-down process The fractionation /cleanup process followed the method reported by Gogou et al. [8]. After the extraction, the DCM solvent was concentrated to incipient dryness by a combination of rotary evaporation and blowing under a gentle nitrogen stream. The concentrated extract was then diluted in $10 \mathrm{ml}$ of $n$-hexane before application to the top of a disposable silica gel (Merck; 0.040-0.063 mm) column. The extract was then fractionated into individual compound classes by flash chromatography on silica gel as follows: The concentrate was applied to the top of a $30 \times 0.7 \mathrm{~cm}$ diameter column, containing $1.5 \mathrm{~g}$ of silica gel (activated at $150^{\circ} \mathrm{C}$ for $3 \mathrm{~h}$ ). Nitrogen pressure was used to obtain a flow of $1.4 \mathrm{ml} \mathrm{min} \mathrm{m}^{-1}$ at the bottom of the column.

2.2.1.3 GC/MS methodology Analyses of all samples were carried out on a Fisons GC800 gas chromatograph interfaced with a Fisons MD800 mass spectrometer (GC/MS) using a DB-5 column $(60 \mathrm{~m} \times 0.25 \mathrm{~mm}$, i.d. $\times 0.25 \mu \mathrm{m}$ film thickness). The ion source temperature, interface temperature and injector port temperature were set at $200^{\circ} \mathrm{C}, 250^{\circ} \mathrm{C}$ and $300^{\circ} \mathrm{C}$ respectively. The carrier gas was helium with a column head pressure of $25 \mathrm{psi}$. The $\mathrm{GC}$ oven temperature

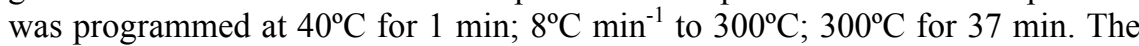
MD800 mass spectrometer operated in selected ion mode. Analytical accuracy and precision - together with internal campaign comparability - was assessed by employing replicate analyses of appropriate standard reference material (i.e. SRM 1944 NIST Marine Sediment).

\subsubsection{OC/EC analysis}

The samples were analyzed for OC and EC using a DRI Model 2001 (Thermal/Optical Carbon Analyzer) with the IMPROVE thermal/optical reflectance (TOR) protocol. The protocol heats a $0.526 \mathrm{~cm}^{2}$ punch aliquot of a sample quartz filter stepwise at temperatures of $120^{\circ} \mathrm{C}(\mathrm{OC} 1), 250^{\circ} \mathrm{C}(\mathrm{OC} 2)$, $450^{\circ} \mathrm{C}$ (OC3), and $550^{\circ} \mathrm{C}$ (OC4) in a non-oxidizing helium atmosphere, and $550^{\circ} \mathrm{C}(\mathrm{EC} 1), 700^{\circ} \mathrm{C}(\mathrm{EC} 2)$, and $800^{\circ} \mathrm{C}(\mathrm{EC} 3)$ in an oxidizing atmosphere of $2 \%$ oxygen in a balance of helium. 


\section{Results and discussion}

\subsection{Estimations of gas-particle partitioning of PAHs}

Predictions of $K_{\mathrm{P}}$ values for PAHs at the Whitbourne site were made using the Dachs-Eisenreich model (Eqn (2)), $K_{\mathrm{OA}}$ model and $K_{\mathrm{SA}}$ model respectively. The calculation used the assumptions that the organic fraction matter $\left(f_{\mathrm{OM}}\right)$ is 2.1 times the organic carbon fraction $\left(f_{\mathrm{OC}}\right)$ and that the ratios of $M W_{\mathrm{OCT}} \gamma_{\mathrm{OCT}} / M W_{\mathrm{OM}} \gamma_{\mathrm{OM}}$ and $a_{\mathrm{Soot}} / a_{\mathrm{EC}}$ used in Eqn (2) are equal to one [9]. Both $K_{\mathrm{OA}}$ model $K_{\mathrm{SA}}$ of PAHs were corrected for the average temperature during the sampling period. Several soot types with specific surface areas of EC $\left(a_{\mathrm{EC}}\right)$ ranging from 3.5 to $761 \mathrm{~m}^{2} \mathrm{~g}^{-1}$ were selected to conduct the sensitivity test of $K_{\mathrm{P}}$ predictions.

\subsection{Role of $\mathrm{OM}$ conversion factor on $K_{\mathrm{P}}$ predictions}

It is well known that both urban and rural aerosols were composed by carbonaceous species such as black carbon (BC) and organic carbon (OC). The limitations of quantitative methods for analyzing OM illustrate that future works is needed on the development of direct measurement. For decades, several attempts have been made to use conversion factor ranging from 1.2 to 2.1 to quantify OM from bulk OC measurements [9]. Based on the current estimation, conversion factor of 1.6 for urban aerosols and 2.1 for rural aerosols seems to be more precise [10]. In this study, the role of OM conversion factor of $K_{\mathrm{P}}$ predictions was investigated by comparing the sensitivity in prediction of $K_{\mathrm{P}}$ with the $\mathrm{OM} / \mathrm{OC}$ ratio of 1.6 and 2.1 as conversion factors for urban and rural aerosols respectively. $K_{\mathrm{P}}$ was estimated by using Eqn (2) and $K_{\mathrm{OA}}$ was predicted by using eqn provided by Odabasi et al. [7].

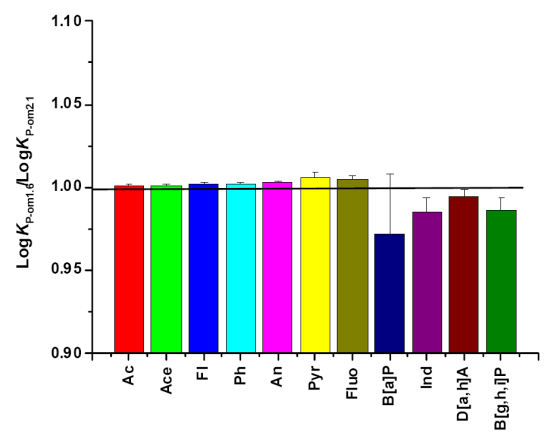

Figure 2: The ratio of $\log K_{\mathrm{P}-\mathrm{OM} 1.6} / \log K_{\mathrm{P}-\mathrm{OM} 2.1}$ of Ac, Ace, Fl, $\mathrm{Ph}, \mathrm{An}, \mathrm{Pyr}$, Fluo, $\mathrm{B}[\mathrm{a}] \mathrm{P}$, Ind, $\mathrm{D}\left[a_{, \mathrm{h}] \mathrm{A} \text { an }} \mathrm{d} \mathrm{B}\left[g_{\mathrm{h}, \mathrm{i}] \mathrm{P}}\right.\right.$ during cold and warm periods in Whitbourne.

Figure 2 shows the ratio of $\log K_{\mathrm{P}-\mathrm{OM} 1.6} / \log K_{\mathrm{P}-\mathrm{OM} 2.1}$ of PAHs based on the $\mathrm{OM}$ conversion factor of 1.6, 2.1 and $a_{\mathrm{EC}}$ of $90 \mathrm{~m}^{2} \mathrm{~g}^{-1}$. The medium MW PAHs 
(i.e. Ph, An, Pyr, Fluo) were in general close to the value of one by $32-53 \%$, while the higher MW PAHs (i.e. B[a]P, Ind, D[a,h]A and $\mathrm{B}[\mathrm{g}, \mathrm{h}, \mathrm{i}] \mathrm{P}$ ) often displayed a higher degree of scatter and the ratio of $\log K_{\mathrm{P}-\mathrm{OM} 1.6} / \log K_{\mathrm{P}-\mathrm{OM} 2.1}$ higher than one by $44-220 \%$ for both seasons. The ratios of $\log K_{\mathrm{P}-\mathrm{OM} 1.6} / \log K_{\mathrm{P} \text { - }}$ OM2.1 of the low MW PAHs (i.e. Ac, Ace, Fl) were in general near the value of one by $24-29 \%$ during cold and warm periods. Based on these results, the predictions of $K_{\mathrm{P}}$ of high MW PAHs were preferentially sensitive to the selection of OM conversion factor, while the predictions of $K_{\mathrm{P}}$ for low MW PAHs were generally more persistent, but still highly significant for medium MW PAHs. As a consequence, some cautions on the selection of OM conversion factor are required for the estimation of $K_{\mathrm{P}}$, especially for $\mathrm{B}[\mathrm{a}] \mathrm{P}$, Ind, $\mathrm{D}[\mathrm{a}, \mathrm{h}] \mathrm{A}$ and $\mathrm{B}[\mathrm{g}, \mathrm{h}, \mathrm{i}] \mathrm{P}$.

\subsection{Role of specific surface area of EC on $K_{\mathrm{P}}$ predictions}

The overall $K_{\mathrm{P}}$ that accounts for both the absorption into organic layer and adsorption on the soot phases is displayed in Eqn (2) with the assumption that there is no distinct difference between soot and atmospheric EC. Dachs and Eisenreich., 2000 used the values of $370 \mathrm{~m}^{2} \mathrm{~g}^{-1}$ and $1,000 \mathrm{~m}^{2} \mathrm{~g}^{-1}$ (i.e. specific surface area of activated carbon) as specific surface areas of elemental carbon $\left(a_{\mathrm{EC}}\right)$ and soot $\left(a_{\mathrm{Soot}}\right)$ respectively. Depending on the adsorptive properties of atmospheric EC, the $K_{\mathrm{P}}$ can be predicted by using $K_{\mathrm{SA}}$ calculated by specific surface area of diesel soot (i.e. NIST standard reference material SRM 1650; 90 $\mathrm{m}^{2} \mathrm{~g}^{-1}$ ). Recently, Arp et al. [11] raised concerns that the adsorption of PAHs on soot surfaces may be a minor of importance because i) the surface area of urban aerosols (e.g., 0.2-2.4 $\mathrm{m}^{2} \mathrm{~g}^{-1}$ ) and road tunnel aerosols (e.g., $7.4 \mathrm{~m}^{2} \mathrm{~g}^{-1}$ ) are 1-2 orders of magnitude smaller than the values for diesel soot (e.g. $90 \mathrm{~m}^{2} \mathrm{~g}^{-1}$ ), and ii) atmospheric EC is coated with salts (e.g. $\left.\left(\mathrm{NH}_{4}\right)_{2} \mathrm{SO}_{4}\right)$ and organics. Therefore, it is important to take these concerns into consideration when one needs to accurately predict $K_{\mathrm{P}}$ of PAHs. In this study, the evaluation of $K_{\mathrm{P}}$ of semi-volatile PAHs, namely $\mathrm{Ph}, \mathrm{An}, \mathrm{Pyr}$ and Fluo, was conducted with different specific areas of atmospheric EC and soot. All values of $a_{\mathrm{EC}}$ and $a_{\text {Soot }}$ used in the present study were reported by Jonker and Koelmans [12]. As illustrated in Table 1, the ratios of $\log K_{\mathrm{P} \text {-prediction }} / \log K_{\mathrm{P} \text {-measurement }}$ for each sampling period were estimated for $\mathrm{Ph}$, An, Pyr and Fluo using three and seven different types of $a_{\text {Soot }}$ and $a_{\mathrm{EC}}$ respectively. With the assumption previously described on atmospheric EC and soot, three scenarios may be considered.

$$
\begin{gathered}
\text { Case I: } \log K_{\text {P-prediction }} / \log K_{\text {P-measurement }}>1 \\
\text { Case II: } \log K_{\mathrm{P} \text {-prediction }} / \log K_{\mathrm{P} \text {-measurement }}=1 \\
\text { Case III: } \log K_{\text {P-prediction }} / \log K_{\text {P-measurement }}<1
\end{gathered}
$$

Case I occurs when the prediction overestimates the measurement as it can be seen from the results of all semi-volatile PAHs using the $a_{\text {Soot }}$ value of activated carbon, regardless of different values of $a_{\mathrm{EC}}$. For instance, the ratios of $\log K_{\mathrm{P}-}$ prediction $/ \log K_{\mathrm{P}-\text { measurement }}$ were higher than one by $31-81 \%$ for both periods. In contrast, much smaller ratios of $\log K_{\text {P-prediction }} / \log K_{\text {P-measurement }}$ were observed for 


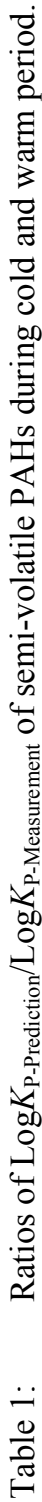

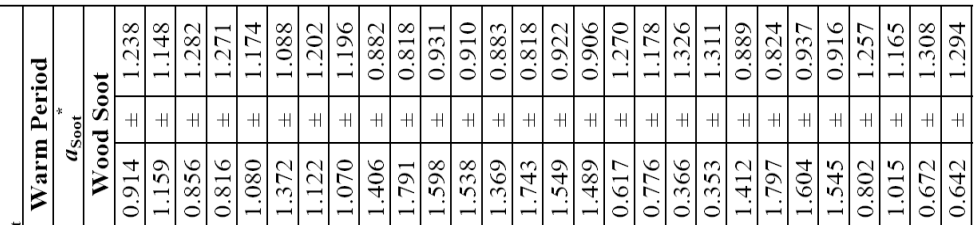

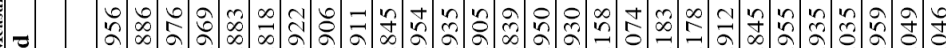

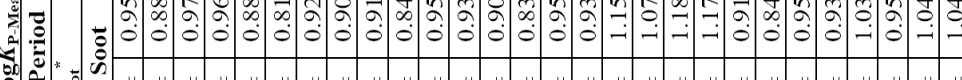
(2)

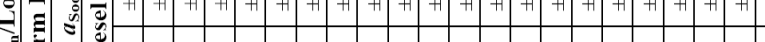

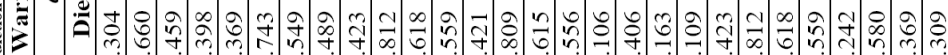

=

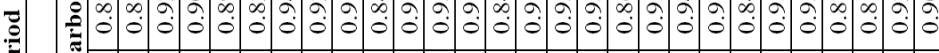
(5)

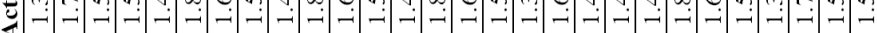

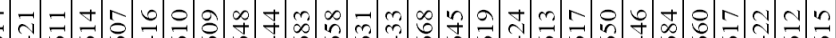

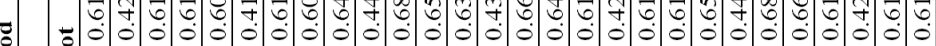
党

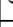

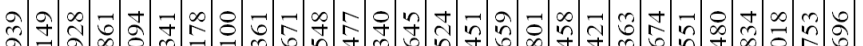

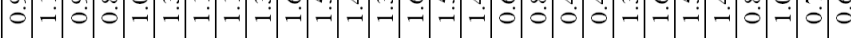

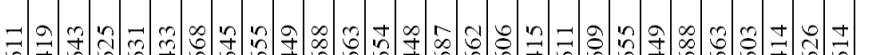

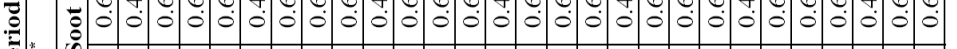
管

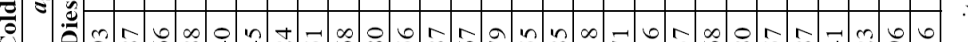

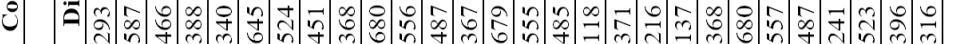

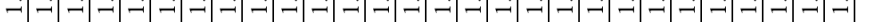

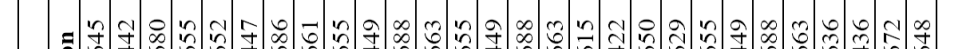

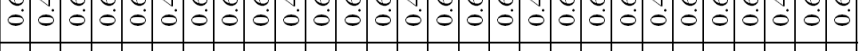

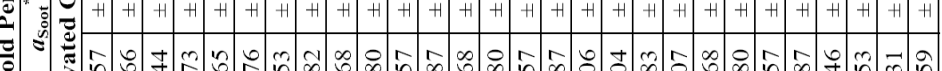

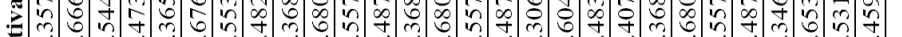
$-\dot{-}-\dot{-}-\dot{-}-\dot{-}-\dot{-}-\dot{-}-\dot{j}-\dot{-}-\dot{-}-\dot{-}-\dot{-}-\dot{-i}-\dot{-}$

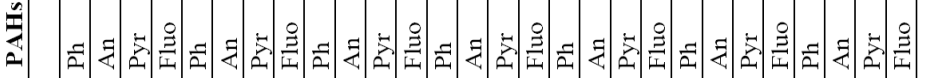

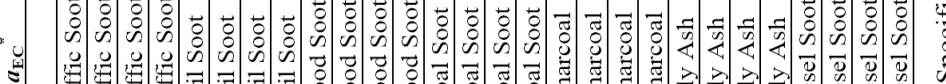

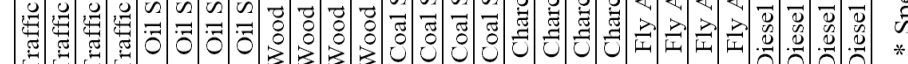


Case III scenario using the specific surface areas of wood soot and charcoal as the values of $a_{\mathrm{Soot}}$ and $a_{\mathrm{EC}}$ respectively. The ratios were $0.66,0.80,0.46,0.42$ and $0.62,0.78,0.37,0.35$ for $\mathrm{Ph}, \mathrm{An}, \mathrm{Pyr}$, Fluo during cold and warm period in that order. Although there was no Case II scenario for all semi-volatile PAHs during both sampling periods, the combination of wood soot and traffic soot as the values of $a_{\text {Soot }}$ and $a_{\mathrm{EC}}$ gave the ratios of $\log K_{\text {P-prediction }} / \log K_{\text {P-measurement }}$ close to one. With this combination, the ratios of $\log K_{\text {P-prediction }} / \log K_{\text {P-measurement }}$ displayed a lower degree of scatter and were lower than one by $6-18 \%$ for both seasons. These results suggest that the reliability in predicting $K_{\mathrm{P}}$ values for semi-volatile PAHs are dependent on the selection of $a_{\text {Soot }}$ and $a_{\mathrm{EC}}$. Hence, some more considerations of sampling location (i.e. aerosol type) are required before selecting the values of $a_{\text {Soot }}$ and $a_{\mathrm{EC}}$.

\subsection{Predictions of $K_{\mathrm{P}}$ of PAHs}

Based on the sensitivity test, the specific areas of wood soot and traffic soot were used as the values of $a_{\mathrm{Soot}}$ and $a_{\mathrm{EC}}$ for the prediction of gas-particle partitioning of PAHs. The OM conversion factor of 2.1 was used in Eqn (2) since this value is considered as suitable for rural aerosol. For the semi-volatile PAHs (Ph, An, Pyr and Fluo), the predictions of partitioning coefficients by the DachsEisenreich model exhibited a good correlation with those of the measured $K_{\mathrm{P}}$ values in both periods (Figure 3). For example, the Dachs-Eisenreich model predicted $K_{\mathrm{P}}$ values of all semi-volatile PAHs a factor of $1.03 \pm 0.12(0.87-$ $1.16)$ and $1.06 \pm 0.15(0.86-1.22)$ of the measured value for cold period and warm period respectively. Conversely, the $K_{\mathrm{OA}}$ based absorptive gas-particle partitioning model invariably predicts lower $K_{\mathrm{P}}$ values $(p<0.001)$ for all semivolatile PAHs with a factor of $0.64 \pm 0.067(0.56-0.72)$ and $0.62 \pm 0.063(0.55$ -0.70 ) of the measured value for cold period and warm period in that order. There was a tendency for the theoretical models to under-predict the values of $\log K_{\mathrm{P}}$. However for some compounds, agreement with the combined adsorption/absorption model was rather good. These compounds were, pyrene, chrysene, benzo(a)anthracene, benzo[a]pyrene, indeno(1,2,3-cd)pyrene and benzo(g,h,i)perylene. A paired $t$-test confirmed the significance of the differences between measured and predicted $K_{\mathrm{P}}$ values $(p<0.001)$ of Ac, Ace, $\mathrm{Fl}, \mathrm{Ph}$ and $\mathrm{An}$, when estimated by all models in both seasons. One explanation is the sampling artifact causing positive biases between low MW PAHs and OC/EC fractions in the measured particulate phase due to adsorption onto the filters. The sorption of gas-phase PAHs to filter surfaces (filter sorption artifact) is known to lead to artificially high measured particulate concentrations and low measured gaseous concentrations, resulting in an artificially high particle-to-gas ratios. It has been widely acknowledged that quartz fiber filters as employed in this study tend to cause positive biases in measured particulate phase concentrations. A fraction of particulate PAH has been proposed to be irreversibly bound to particles in the atmosphere although it can be extracted by standard analytical methods. Any PAH subject to the effect of "non-exchangeability" of bound material would be likely to have a higher $\log K_{\mathrm{P}}$ value than that calculated for 


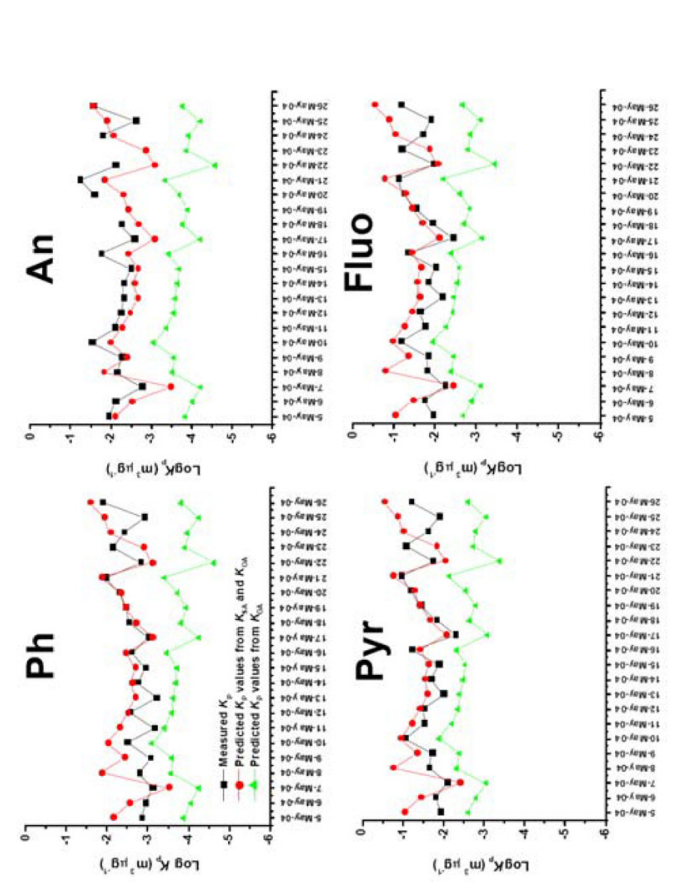

矛这

ฮี

อ

बiv

论

결

료

$\stackrel{0}{\frac{\pi}{3}}$

老岤

్ㅠㅇ

들.응

它

$\cdot \stackrel{\oplus}{\oplus}$

$\stackrel{\square}{\Xi}$

芯

$\mathscr{E}$

宅:

声 $\frac{\dot{0}}{3}$
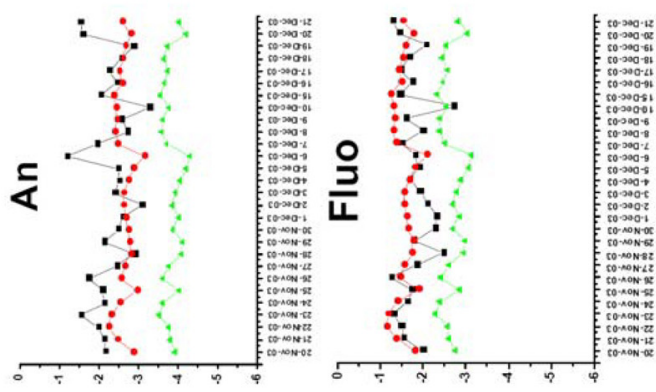

$\mp$ (

武艺

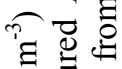

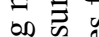

先 胥

䒔

फิำ

\&

敢

更总

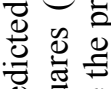
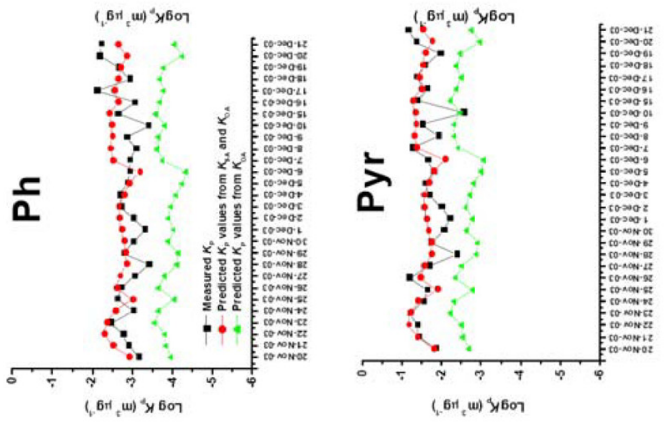

记艺

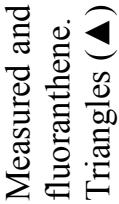

$\ddot{m}$ 
equilibrium partitioning in the atmosphere. Even a small fraction of nonexchangeable particulate PAHs is significant in the case of low MW PAH and thus the "non-exchangeability" effect may be another cause of discrepancy between the measured and predicted $\log K_{\mathrm{p}}$ of highly volatile PAHs.

\subsection{Seasonal effects on gas-particle partitioning mechanism}

There was a large seasonal difference in the total (vapor plus particulate) concentration of the PAH. This is consistent with the potential for better dispersion of primary emissions during the warm period months and also greater decomposition by chemical reactions. However, one other observation which was not anticipated was that there is little difference in vapor to particle partitioning (as represented by $\log K_{\mathrm{P}}$ ) between the seasons and for phenanthrene and heavier MW compounds there is no significant difference between $\log K_{\mathrm{P}}$ in warm period and cold period. This is seen clearly in Figure 3 and must result from a difference in the $a b$ sorptive/adsorptive properties of the particles between the seasons. Recent work by Ribes et al. (2003) has demonstrated that the ratio of $f_{\mathrm{EC}} K_{\mathrm{SA}} \gamma_{\mathrm{OCT}} / f_{\mathrm{OM}} K_{\mathrm{OA}}$ can be used as an indicator to investigate the gas-particle partitioning processes. This ratio will allow clarification of the prevalent mechanisms for gas-particle partitioning of PAHs. Dachs and Eisenreich [5] proposed that the mechanisms governing gas-particle partitioning of PAHs can be sub-divided into three scenarios or cases according to the ratio of $f_{\mathrm{EC}} K_{\mathrm{SA}} \gamma_{\mathrm{OCT}} / f_{\mathrm{OM}} K_{\mathrm{OA}}$. When the ratio of $f_{\mathrm{EC}} K_{\mathrm{SA}} \gamma_{\mathrm{OCT}} / f_{\mathrm{OM}} K_{\mathrm{OA}}>5$, the gas-particle partitioning will be dominated by adsorption onto the soot phase ( $K_{\mathrm{SA}}$ model), whereas the ratio of $f_{\mathrm{EC}} K_{\mathrm{SA}} \gamma_{\mathrm{OCT}} / f_{\mathrm{OM}} K_{\mathrm{OA}}<0.2$ represents for the $a b$ sorption into organic matter $\left(K_{\mathrm{OA}}\right.$ model) and values between 0.2 and 5 occur when both $a b$ sorption into organic layer and $a d$ sorption onto the soot carbon are important (i.e. Dachs-Eisenreich model). At the Whitbourne site, the ratio of $f_{\mathrm{EC}} K_{\mathrm{SA}} \gamma_{\mathrm{OCT}} / f_{\mathrm{OM}} K_{\mathrm{OA}}$ ranged from 0.20 to 3.9 with the average of $0.86 \pm 0.54$ and $2.09 \pm 1.20$ for cold period and warm period respectively. All PAHs studied correspond to a $f_{\mathrm{EC}} K_{\mathrm{SA}} \gamma_{\mathrm{OCT}} / f_{\mathrm{OM}} K_{\mathrm{OA}}$ ratio in the range between 0.2 and 5 and thus one can conclude that both adsorption and absorption influence gas-particle partitioning of PAHs in Whitbourne for both seasons. In contrast, it has been observed that absorption into organic matter may account for less than $10 \%$ of the total PAHs in the Chesapeake Bay particulate phase. Some studies reported that $a d$ sorption and desorption of PAHs to and from combustion aerosols may take hours to reach equilibrium, possibly due to the occurrence of a liquid-like organic film coating the elemental carbon. Thus the mass transfer rate of PAHs is limited by the diffusion between the liquid-like organic phase and elemental carbon. A paired $t$-test was conducted to test the statistical differences of averaged $f_{\mathrm{EC}} K_{\mathrm{SA}} \gamma_{\mathrm{OCT}} / f_{\mathrm{OM}} K_{\mathrm{OA}}$ ratios between the two seasons. For low and medium MW PAHs, there were no statistical differences $(p<0.05)$ in seasonality. However, the seasonal differences of $f_{\mathrm{EC}} K_{\mathrm{SA}} \gamma_{\mathrm{OCT}} / f_{\mathrm{OM}} K_{\mathrm{OA}}$ ratios are statistically significant $(p<0.05)$ for high MW PAHs. Since unusually the average $f_{\mathrm{EC}}$ value of warm period aerosols was higher than that of cold period aerosols, the higher average $f_{\mathrm{EC}} K_{\mathrm{SA}} \gamma_{\mathrm{OCT}} / f_{\mathrm{OM}} K_{\mathrm{OA}}$ ratios in warm period are largely attributable to the relatively high abundances of EC. This is related to the back 
trajectories which were predominantly maritime air in cold period, but contained a number of continental European air masses in warm period which would have received substantial emissions from diesel vehicles.

\section{Conclusions}

Air samples were collected at the rural site of Whitbourne in the UK and the seasonal variation of the gas-particle partitioning coefficient $\left(K_{\mathrm{P}}\right)$ of PAHs was studied. The estimation of $K_{\mathrm{P}}$ by three different models, namely DachsEisenreich, $K_{\mathrm{OA}}$ and $K_{\mathrm{SA}}$ models reveals that the inclusion of both adsorption and absorption mechanisms substantially improves the prediction accuracy. The results of model analysis of selected PAH congeners have shown that no model was very suitable for the prediction of $K_{\mathrm{P}}$ for low MW PAHs. The discrepancy between predicted and measured $K_{\mathrm{P}}$ in low MW PAHs may be caused by several factors including i) sampling artefacts, ii) the effect of "non-exchangeability", iii) uncertainties in calculation of $K_{\mathrm{OA}}$ and $K_{\mathrm{SA}}$, iv) variability of the activity coefficients in polar aerosol.

\section{Acknowledgements}

The authors thank Mr Patrick and Mrs Kristine Evans for their contribution in providing the facility to collect data and National Center of Excellence for Environmental and Hazardous Waste Management-PSU Satellite Center, Prince of Songkla University for financial support (to S.P.). British Atmospheric Data Center (BADC) supported this work through 24-h back trajectory analysis.

\section{References}

[1] Korenaga, T., Liu, X.X and Huang, Z.Y., The influence of moisture content on polycyclic aromatic hydrocarbons emission during rice straw burning. Chemosphere - Global Change Science, 3, pp.117-122, 2001.

[2] IARC, 1987. Overall evaluations of carcinogenicity: An updating of IARC monographs vols. 1 to 42 . Monographs on the evaluation of carcinogenic risks to humans, Suppl. 7. International Agency for Research on Cancer, Lyon.

[3] Poor, N., Tremblay, R., Kay, H., Bhethanabotla, V., Swartz, E., Luther, M and Campbell, S., Atmospheric concentrations and dry deposition rates of polycyclic aromatic hydrocarbons (PAHs) for Tampa Bay, Florida, USA, Atmospheric Environment, 38, pp. 6005-6015, 2004.

[4] Sitaras, I.E., Bakeas, E.B and Siskos, P.A., Gas/particle partitioning of seven polycyclic aromatic hydrocarbons in a heavy traffic urban area. The Science of the Total Environment, 327, pp. 249-264, 2004.

[5] Dachs, J and Eisenreich, S.J., Adsorption onto aerosol soot carbon dominates gas-particle partitioning of polycyclic aromatic hydrocarbons. Environmental Science and Technology, 34, pp.3690-3697, 2000. 
[6] Fenizio, A., Mackay, D., Bidleman, T and Harners, T., Octanol-air partition coefficient as a predictor of partitioning of semi-volatile organic chemicals to aerosols. Atmospheric Environment, 31(15), pp. 2289-2296, 1997.

[7] Odabasi, M., Cetin, E and Sofuoglu, A., Determination of octanol-air partition coefficients and supercooled liquid vapor pressures of PAHs as a function of temperature: Application to gas-particle partitioning in an urban atmosphere. Atmospheric Environment, 40(34), pp.6615-6625, 2006.

[8] Gogou, A. I., Apostolaki M and Stephanou. G. E., Determination of organic molecular markers in marine aerosols and sediments: one-step flash chromatography compound class fractionation and capillary gas chromatographic analysis. Journal of Chromatography A, 799, pp.215-231, 1997.

[9] Countess, R.J., Wolff, G.T and Cadle, S.T., The Denver winter aerosol: a comprehensive chemical characterization, Air Pollution Control Association, 30, pp.1194-1200, 1980.

[10] Turpin, B. J and Lim, H. J., Species contribution to PM2.5 mass concentrations: Revisiting common assumptions for estimating organic mass. Aerosol Science and Technology, 35, pp.602-610, 2001.

[11] Jonker, M.T.O and Koelmans, A.A., Sorption of polycyclic aromatic hydrocarbons and polychlorinated biphenyls to soot and soot-like materials in the aqueous environment: mechanistic considerations. Environmental Science and Technology, 36, pp. 3725-3734, 2002.

[12] Arp, P.H., Schwarzenbach, R.P and Goss, K.U., Ambient Gas/Particle Partitioning. 1. Sorption Mechanisms of Apolar, Polar, and Ionizable Organic Compounds. Environmental Science and Technology, 42 (15), pp. 5541-5547, 2008. 